\title{
Tenofovir alafenamide nephrotoxicity: a case report and literature review
}

\author{
Thornthun Ueaphongsukkit 1,2, Sivaporn Gatechompol2,3, Anchalee Avihingsanon 2,3, Jerasit Surintrspanont ${ }^{4}$, \\ Kroonpong lampenkhae ${ }^{4}$, Yingyos Avihingsanon ${ }^{5,6,7}$ and Suwasin Udomkarnjananun ${ }^{5,6,7^{*}}$ (D)
}

\begin{abstract}
Background: Tenofovir alafenamide (TAF), a novel prodrug of tenofovir (TFV), has become the preferred drug for the treatment of HIV-1 and chronic hepatitis B infection in clinical practice. Results from clinical trials showed that it had better renal and bone mineral outcomes compared to tenofovir disoproxil fumarate (TDF). However, as we have seen with TDF, side effects from the new medication can be more prevalent and recognized after extensive use in real world situations. Sporadic cases of acute kidney injury in patients using TAF have started to emerge.

Case presentation: We report a case of 49-year-old Thai, HIV treatment-experienced female with hypertension presented with worsening renal function after switching her antiretroviral regimen from TDF, emtricitabine (FTC), and lopinavir/ritonavir (LPV/r) to TAF, FTC and dolutegravir (DTG) for 3 months. Kidney biopsy showed distinctive picture of tenofovir nephrotoxicity with acute tubular injury and mitochondrial injury. The possible causes of acute kidney injury and nephrotoxicity from TAF for this patient were discussed. We have extensively reviewed all published case reports of TAF-associated nephrotoxicity and summarized the essential information in this article.
\end{abstract}

Conclusion: Although TAF has less nephrotoxicity compared with TDF; renal function should always be monitored after the initiation of both drugs. Future large cohort studies are required to identify the risk factors of TAF-associated nephrotoxicity and to design an effective preventive strategy.

Keywords: Tenofovir alafenamide, Acute kidney injury, Nephrotoxicity, Renal pathology, Mitochondria, HIV, Antiretroviral therapy, Case report

\section{Introduction}

Tenofovir (TFV) has become one of the backbone antiretroviral therapies (ART) in this era. However, the nephrotoxicity profile which is caused by cytoplasmic and intra-mitochondrial accumulation of TFV and results in the messenger ribonucleic acid (mRNA) depletion, mitochondrial deoxyribonucleic acid (DNA) depletion, and oxidative respiratory chain dysfunction, eventually contribute to proximal renal tubular abnormalities and

\footnotetext{
*Correspondence: suwasin.u@gmail.com

${ }^{5}$ Division of Nephrology, Department of Medicine, Faculty of Medicine,

Chulalongkorn University and King Chulalongkorn Memorial Hospital,

1873, Rama 4 road, Pathumwan, 10330 Bangkok, Thailand

Full list of author information is available at the end of the article
}

renal insufficiency, limit its use in clinical practice $[1,2]$. Tenofovir alafenamide (TAF) is a novel prodrug of TFV. It has a more favorable renal and bone safety profiles than its predecessor tenofovir disoproxil fumarate (TDF). Since 2015, TAF was approved by the U.S. Food and Drug Administration (FDA) as the first-line treatment of HIV in adults and adolescents. It is recommended as the preferred nucleotide analogue reverse transcriptase inhibitor (NRTI) backbone of the ART in the current HIV treatment guidelines [3, 4]. Since TAF has become more widely available, sporadic cases of acute kidney injury in patients using TAF is increasing [5-9]. Here we present a patient with TAF-containing ART regimen who came to our hospital with kidney injury. original author(s) and the source, provide a link to the Creative Commons licence, and indicate if changes were made. The images or other third party material in this article are included in the article's Creative Commons licence, unless indicated otherwise in a credit line to the material. If material is not included in the article's Creative Commons licence and your intended use is not permitted by statutory regulation or exceeds the permitted use, you will need to obtain permission directly from the copyright holder. To view a copy of this licence, visit http://creativecommons.org/licenses/by/4.0/. The Creative Commons Public Domain Dedication waiver (http://creativeco mmons.org/publicdomain/zero/1.0/) applies to the data made available in this article, unless otherwise stated in a credit line to the data. 


\section{Case presentation}

The patient was a 49-year-old Thai female with HIV infection, hypertension, and dyslipidemia. She was diagnosed with HIV infection since 1997 and had been exposed to multiple antiretroviral medications. In October 2001, she finally achieved undetectable viral load ( $<50$ copies $/ \mathrm{mL})$. In November 2015, she started to use TDF-based ART, which was TDF, emtricitabine (FTC), and lopinavir/ritonavir $(\mathrm{LPV} / \mathrm{r})$. Her serum creatinine and estimated glomerular filtration rate (eGFR) by CKD-EPI were stable at $<1 \mathrm{mg} / \mathrm{dL}$ and $>80 \mathrm{ml} / \mathrm{min} / 1.73 \mathrm{~m}^{2}$ since then.

In October 2019, her regimen was switched to a once daily, fixed dose combination pill containing TAF $25 \mathrm{mg}$, FTC $200 \mathrm{mg}$, and dolutegravir (DTG) $50 \mathrm{mg}$ (TAF/FTC/ DTG). Three months after she had changed her regimen (January 2020), serum creatinine increased from baseline of $1.05 \mathrm{mg} / \mathrm{dL}$ to $1.47 \mathrm{mg} / \mathrm{dL}$. At 6 months follow-up (April 2020), her serum creatinine continuously increased to $2.30 \mathrm{mg} / \mathrm{dL}$ which prompted further investigation as described below.

Other concomitant medications included amlodipine $5 \mathrm{mg} /$ day for hypertension and atorvastatin $20 \mathrm{mg} /$ day for dyslipidemia. However, in January 2020, her hypertension was not well controlled and amlodipine was increased to $10 \mathrm{mg} /$ day.

\section{Investigations}

The patient's baseline serum creatinine was within 0.8$1.0 \mathrm{mg} / \mathrm{dL}$ and eGFR (CKD-EPI) was within $70-85 \mathrm{~mL} /$ $\min / 1.73 \mathrm{~m}^{2}$ as shown in Fig. 1. Retrospective review of the medical record showed that she had persistent red blood cells $(\mathrm{RBC})$ within the range of $3-5$ cells/high power field (HPF) in urine and $1+$ proteinuria for nearly eight years.

After starting of TAF/FTC/DTG, her serum creatinine was rapidly increased to $2.30 \mathrm{mg} / \mathrm{dL}$ and eGFR declined to $24 \mathrm{~mL} / \mathrm{min} / 1.73 \mathrm{~m}^{2}$. Creatine phosphokinase (CPK) was within the normal range. Ultrasonography revealed a mild dilatation at the left pelvicalyceal system and mild hydronephrosis which could not be responsible for the significant deterioration of the patient's renal function.

A kidney biopsy was then performed. From the light microscopic examination of the specimen, glomeruli with mild mesangial expansion and mesangial hypercellularity were detected (Fig. 2A). Some tubules showed apical blebs and disrupted brush border (Fig. 2B). Apoptotic tubular cells were occasionally seen (Fig. 2B). Eosinophilic intracytoplasmic inclusions in the proximal tubular epithelial cells resembled to megamitochondria were identified in a few tubules (Fig. 2C). Immunofluorescence study showed granular staining of IgA $3+$ and C3 $1+$ in the mesangium. The pathological findings were compatible with IgA nephropathy (Oxford classification M1 E0 S1 T1 C0) with acute tubular injury suspected to be from drug toxicity (tenofovir).

\section{Treatment and outcome}

Since the patient's renal function continuously declined, TAF/FTC/DTG was discontinued. Lopinavir/ritonavir and low dose lamivudine were prescribed thereafter. After TAF/FTC/DTG was discontinued for 2 months, her serum creatinine decreased to $1.82 \mathrm{mg} / \mathrm{dL}$ and

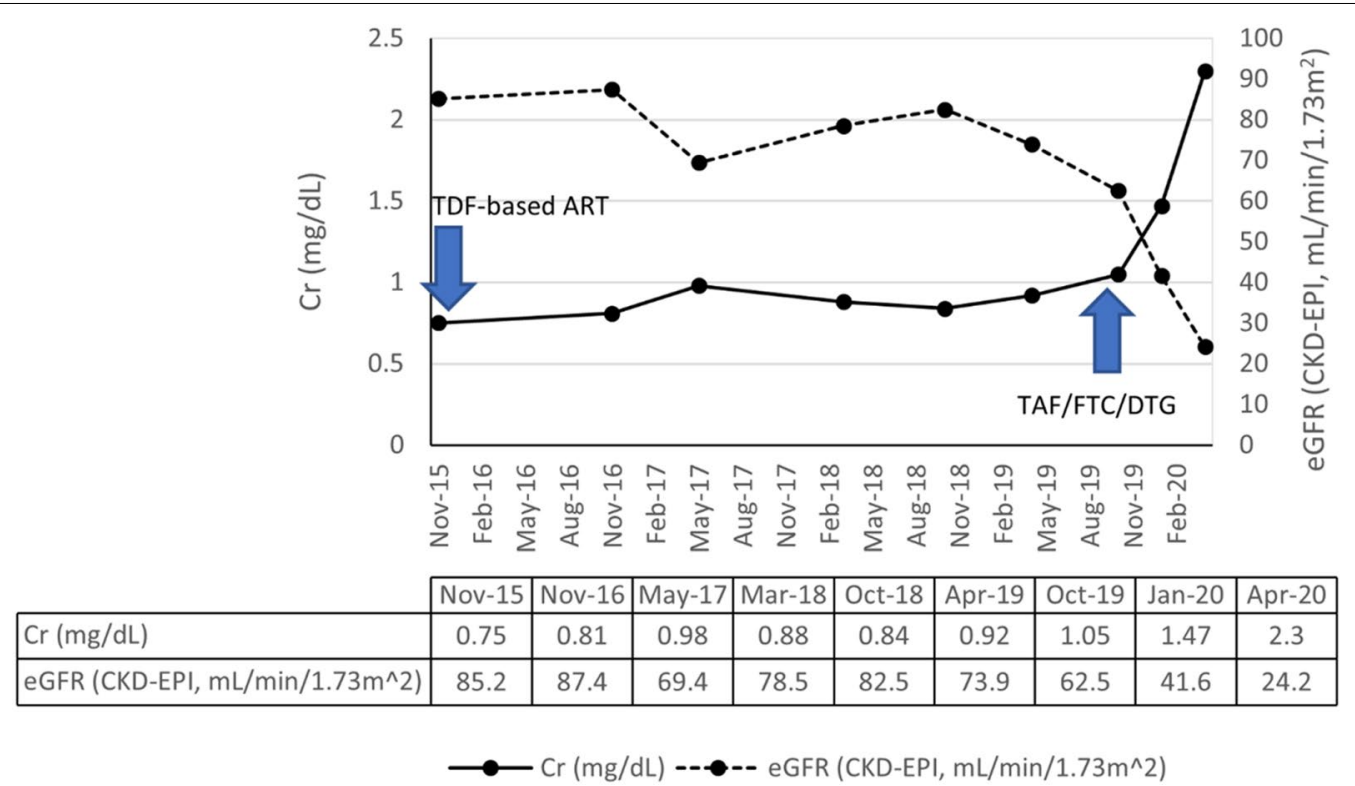

Fig. 1 The patient's serum creatinine and eGFR (CKD-EPI creatinine equation) before and after starting TAF/FTC/DTG 


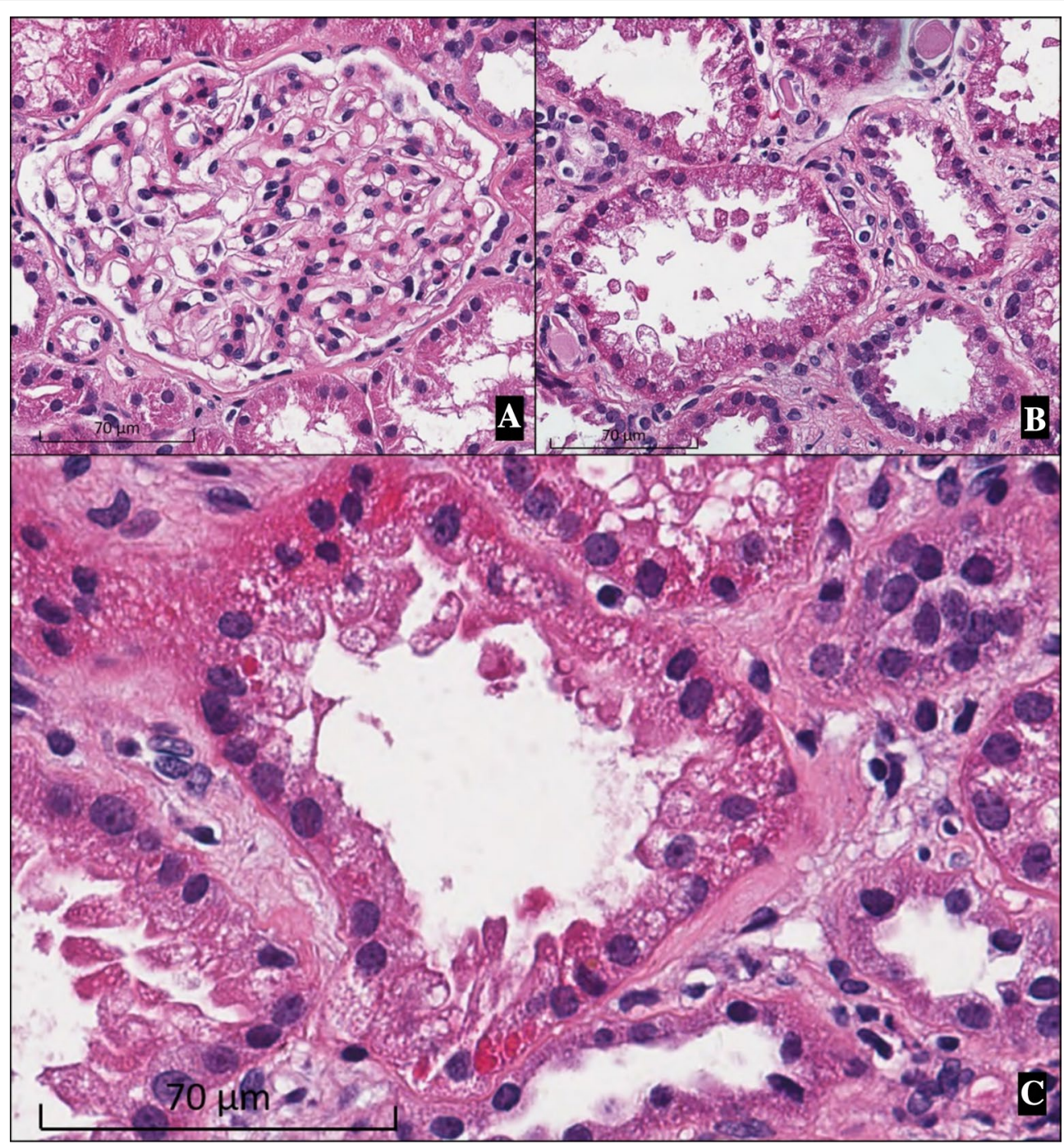

Fig. 2 Kidney biopsy (hematoxylin and eosin staining, original magnification $\times$ 400). A A glomerulus with mild mesangial expansion and mesangial hypercellularity. B Acute tubular injury showing apical blebs, disruption of the brush border, apoptotic and sloughing of the renal tubular epithelial cells. C Megamitochondria is suspected from eosinophilic intracytoplasmic inclusions in the proximal tubular epithelial cells

eGFR improved from 24 to $32 \mathrm{~mL} / \mathrm{min} / 1.73 \mathrm{~m}^{2}$. Her renal function was stabilized within the eGFR range of $35-40 \mathrm{~mL} / \mathrm{min} / 1.73 \mathrm{~m}^{2}$ during the next 6 months. The patient had no hypophosphatemia or hypokalemia. After renal function was steady, amlodipine was switched to losartan to reduce proteinuria and control the blood pressure. Patient's proteinuria was stable at $1.5-2 \mathrm{~g} /$ day during the follow-up periods after the initiation of losartan. Urine RBCs was persistently positive for 1-5 cells/HPF. After discussed with the patient regarding eGFR, proteinuria, and hypertension, kidney biopsy will be reperformed if these parameters are worsened, and corticosteroid is planned if there is a significant progression of IgA nephropathy.

\section{Discussion}

This article presented a case report of the HIV-infected patient who had an acute kidney injury while receiving TAF/FTC/DTG. Her renal function had been stable for many years with the TDF-containing ART, before switching to the culprit regimen. Many possibilities are needed to be discussed of how her renal function was deteriorated.

First, the pathological findings suggested that the patient had a significant acute tubular injury which could have been caused by tenofovir toxicity. Tenofovir (TFV)associated nephrotoxicity have long been described. It is more well-known with TDF which is a prodrug of TFV. TDF is converted to TFV in plasma and can damage the 
mitochondria of the proximal tubular cells [10]. Conversely, TAF is another TFV prodrug that is more stable in the plasma and is converted to TFV intracellularly [11]. Previous studies showed that therapeutic dose of TAF has a better renal safety profile compared with TDF, due to the fact that the level of TFV in the plasma is lower, hence lessens the exposure of proximal tubular epithelial cells to TFV [12-14]. However, TFV from TAF is still renally excreted and may cause tubular injury [15-17]. A pharmacokinetic study of a single-dose TAF administered to severe renal impairment participants with eGFR $15-29 \mathrm{~mL} / \mathrm{min} / 1.73 \mathrm{~m}^{2}$ resulted in higher TAF and TFV plasma levels compared to the matched normal healthy controls with eGFR $\geq 90 \mathrm{~mL} / \mathrm{min} / 1.73 \mathrm{~m}^{2}$ [18]. As a result, one can postulate that in patients with baseline renal insufficiency, the regular dose of TAF could result in higher plasma TFV level and lead to higher risk of kidney injury, compared with the normal eGFR patients. Case reports of TAF-associated nephrotoxicity were reviewed and shown in Table 1. [5-9]. Most of the cases had either underlying renal abnormalities or concurrent use of nephrotoxic medications. These renal diseases and co-medications possibly put the patients at risk of TFV-associated nephrotoxicity by decreasing TFV renal clearance [16]. In addition, renal diseases and medications could also be the causes of acute kidney injury by themselves. Our patient might have an underlying stable IgA nephropathy for a long period which did not display any problem while TDF had been used. Moreover, TDF was stopped for 3 months prior to the presentation and 8 months before the biopsy, while TAF was used at the time of presentation. Hence, this information supports the hypothesis that TAF, rather than TDF, were the cause of acute tubular injury in this case. However, we cannot completely exclude the possibility that the histopathology of TFV-associated nephrotoxicity was partly contributed from TDF before replaced by TAF [19, 20]. However, TDF solely cannot explain the clinical course of acute kidney injury in this patient.

Second, DTG can inhibit organic cation transporter 2 (OCT2) at the basolateral membrane of the proximal renal tubular cells which mediates tubular uptake of creatinine, results in decreasing of creatinine clearance and rising of serum creatinine without changing the true glomerular filtration rate [21-23]. From previous studies, estimated decrease in creatinine clearance was approximately $10-14 \%$ from baseline, and increase of serum creatinine of less than $0.5 \mathrm{mg} / \mathrm{dL}$ [21, 24-27]. The serum creatinine are usually stabilized after $2-4$ weeks of DTG initiation [25-27]. It is possible that DTG could partly contribute to the rising of serum creatinine in this patient. However, her renal function continued to decline beyond the threshold of DTG effect, thus it is unlikely that DTG was the sole cause of an acute kidney injury. However, there are still limited information of the pharmacokinetic and drug interaction between DTG and TAF in the Asian population. In a large randomized controlled trial of DTG and TAF conducted in African patients with creatinine clearance higher than $60 \mathrm{~mL} / \mathrm{min}$ showed that the combination of DTG and TAF had minimal adverse effects [28]. However, pharmacogenomic of DTG and TAF in Asians might be different from Africans. It is possible that this combination might increase the plasma and intracellular TFV to the toxic levels. Additional pharmacokinetics studies of DTG in combination with TAF in Asian population are needed.

IgA nephropathy could have caused the patient's abnormal urinary sediments and proteinuria for many years before this presentation. IgA nephropathy is the most common primary glomerular disease in Asia and has been associated with various inflammatory and infectious diseases, including HIV infection [29, 30]. This patient might develop IgA nephropathy since 2012 when she started having microscopic hematuria and proteinuria. The pathological findings based on the Oxford (MEST) classification; mesangial hypercellularity (M1), segmental glomerulosclerosis (S1), and tubular atrophy and interstitial fibrosis (T1) were all described as poor prognostic markers for IgA nephropathy [31-35]. Thus, IgA nephropathy could be partly responsible to renal function deterioration in our case. However, the improvement and stabilization of renal function after TAF discontinuation and before the initiation of losartan cannot be exclusively explained by IgA nephropathy.

There are also some limitations in our report. Although there was a pathological evidence of tubular injury from tenofovir from the kidney biopsy, our case did not have other laboratory abnormalities associated with proximal renal tubulopathy such as glucosuria, phosphaturia, hypophosphatemia, hypokalemia, or metabolic acidosis. However, certain group of patients can have tenofovirassociated nephrotoxicity without classical laboratory abnormalities as previously been reported $[10,36]$. Since the kidney pathology showed both the characteristics of IgA nephropathy and tenofovir-nephrotoxicity, it is worth to keep in mind that the combination of both, rather than the single entity, had an effect on the patient's renal function.

In conclusion, the temporal relationship of the event in our case suggested that the cause of acute kidney disease could be multifactorial. However, the evidence from kidney biopsy informed that the possibility of TAF-associated nephrotoxicity could not be omitted. Clinicians should be aware of this adverse effect of TAF, especially when the drug is prescribed in patients with an underlying renal disease. In these patients, renal 


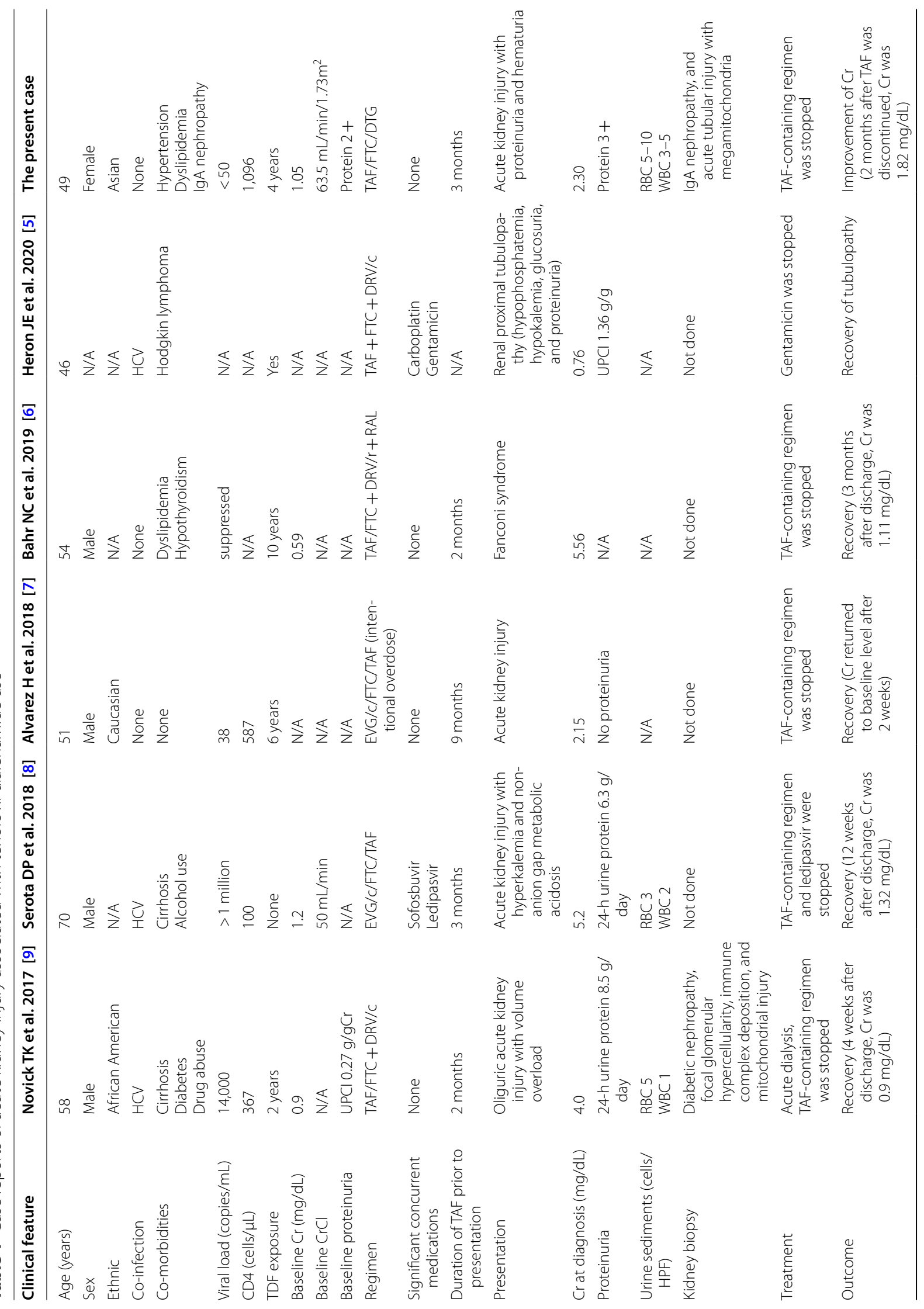




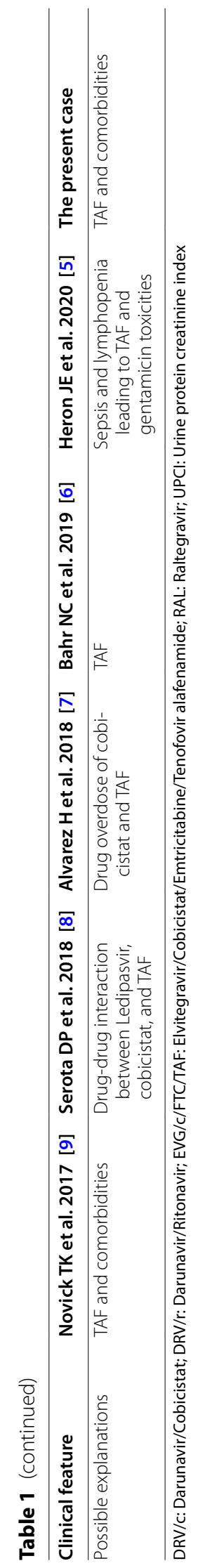


function should be closely monitored after the initiation of TAF. Literatures review of TAF-associated nephrotoxicity are summarized in Table 1 , which the general prognosis was good and renal function could spontaneously recover after the withdrawal of the medication.

\section{Abbreviations}

ART: Antiretroviral therapy; Cr: Creatinine; $\mathrm{CrCl}$ : Creatinine clearance; CPK: Creatine phosphokinase; DRV/c: Darunavir/Cobicistat; DRV/r: Darunavir/Ritonavir; DTG: Dolutegravir; EFV: Efavirenz; eGFR: Estimated glomerular filtration rate; EVG/C/FTC/TAF: Elvitegravir/Cobicistat/Emtricitabine/Tenofovir alafenamide; FTC: Emtricitabine; HPF: High power field; LPV/r: Lopinavir/ritonavir; OCT2: Organic cation transporter 2; RAL: Raltegravir; RBC: Red blood cells; TAF: Tenofovir alafenamide; TDF: Tenofovir disoproxil fumarate; TFV: Tenofovir; UPCI: Urine protein creatinine index; WBC: White blood cells.

\section{Acknowledgements}

The authors would like to thank June Ohata for English editing of the manuscript. The authors thank to Tuberculosis Research Unit, Faculty of medicine, Chulalongkorn University for providing the support in this study.

\section{Authors' contributions}

TU prepared the first draft of this manuscript. JS, KI, and SU reviewed the pathology of the specimens and provided images for the manuscript. SG, AA, $Y A$, and SU reviewed and edited the manuscript. Each author contributed important intellectual content during manuscript drafting or revision and accepts accountability for the overall work by ensuring that questions pertaining to the accuracy or integrity of any portion of the work are appropriately investigated and resolved. All authors have read and approved the final manuscript.

\section{Funding}

TU is supported by Second Century Fund (C2F) for post-doctorate researcher, Chulalongkorn University. Funders had no role in study design; collection, analysis, and interpretation of data; writing the report; and the decision to submit the report for publication.

\section{Availability of data and materials}

All data are presented in the manuscript. Additional data are available on reasonable request.

\section{Declarations}

\section{Ethical approval and consent to participate}

This study was approved by the Ethical Committee of the Faculty of Medicine, Chulalongkorn University, in compliance with the international guidelines for human research protection as Declaration of Helsinki (IRB No. 161/45).

\section{Consent to publication}

The authors declare that they have obtained a consent from the patient for the publication of academic knowledge.

\section{Competing interests}

AA has received honorarium for consultation from ViiV Healthcare. The rest of the authors declare no relevant financial interests.

\section{Author details}

'Department of Medicine, Faculty of Medicine, Chulalongkorn University, Bangkok, Thailand. ${ }^{2}$ The HIV Netherlands Australia Thailand Research Collaboration (HIV-NAT), Thai Red Cross AIDS Research Centre, Bangkok, Thailand. ${ }^{3}$ Tuberculosis Research Unit, Faculty of Medicine, Chulalongkorn University, Bangkok, Thailand. ${ }^{4}$ Department of Pathology, Faculty of Medicine, Chulalongkorn University, Bangkok, Thailand. ${ }^{5}$ Division of Nephrology, Department of Medicine, Faculty of Medicine, Chulalongkorn University and King Chulalongkorn Memorial Hospital, 1873, Rama 4 road, Pathumwan, 10330 Bangkok, Thailand. ${ }^{6}$ Excellence Center for Solid Organ Transplantation, King
Chulalongkorn Memorial Hospital, Bangkok, Thailand. 'Renal Immunology and Transplant Research Unit, Chulalongkorn University, Bangkok, Thailand.

Received: 7 January 2021 Accepted: 12 August 2021

Published online: 21 August 2021

\section{References}

1. Tourret J, Deray G, Isnard-Bagnis C. Tenofovir effect on the kidneys of HIV-infected patients: a double-edged sword? J Am Soc Nephrol. 2013;24(10):1519-27.

2. Fernandez-Fernandez B, Montoya-Ferrer A, Sanz AB, et al. Tenofovir nephrotoxicity: 2011 update. AIDS Res Treat. 2011;2011:354908.

3. Saag MS, Benson CA, Gandhi RT, et al. Antiretroviral drugs for treatment and prevention of HIV infection in adults: 2018 recommendations of the International Antiviral Society-USA panel. JAMA. 2018;320(4):379-96.

4. Ryom L, Cotter A, De Miguel R, et al. EACS Governing Board. 2019 update of the European AIDS Clinical Society Guidelines for treatment of people living with HIV version 10.0. HIV Med. 2020 Nov;21(10):617-624.

5. Heron JE, Bloch M, Vanguru V, et al. Renal proximal tubulopathy in an HIV-infected patient treated with tenofovir alafenamide and gentamicin: a case report. BMC Nephrol. 2020;21(1):339.

6. Bahr NC, Yarlagadda SG. Fanconi syndrome and tenofovir alafenamide: a case report. Ann Intern Med. 2019;170(11):814-5.

7. Alvarez H, Marino A, Valcarce N, et al. Overdose of elvitegravir/cobicistat/ emtricitabine/tenofovir alafenamide in an HIV-1-infected subject with attempted suicide. Infection. 2019;47(1):115-9.

8. Serota DP, Franch HA, Cartwright EJ. Acute kidney injury in a patient on tenofovir alafenamide fumarate after initiation of treatment for hepatitis C virus infection. Open Forum Infect Dis. 2018;5(8):ofy189.

9. NovickTK, Choi MJ, Rosenberg AZ, et al. Tenofovir alafenamide nephrotoxicity in an HIV-positive patient: a case report. Medicine (Baltimore). 2017;96(36):e8046.

10. Herlitz LC, Mohan S, Stokes MB, et al. Tenofovir nephrotoxicity: acute tubular necrosis with distinctive clinical, pathological, and mitochondrial abnormalities. Kidney Int. 2010;78(11):1171-7.

11. Atta MG, De Seigneux S, Lucas GM. Clinical pharmacology in HIV therapy. Clin J Am Soc Nephrol. 2019;14(3):435-44.

12. Ray AS, Fordyce MW, Hitchcock MJ. Tenofovir alafenamide: a novel prodrug of tenofovir for the treatment of Human Immunodeficiency Virus. Antiviral Res. 2016;125:63-70.

13. Post FA, Tebas P, Clarke A, et al. Brief report: switching to tenofovir alafenamide, coformulated with elvitegravir, cobicistat, and emtricitabine, in HIV-infected adults with renal impairment: 96-week results from a single-arm, multicenter, open-label phase 3 study. J Acquir Immune Defic Syndr. 2017;74(2):180-4.

14. Gupta SK, Post FA, Arribas JR, et al. Renal safety of tenofovir alafenamide vs. tenofovir disoproxil fumarate: a pooled analysis of 26 clinical trials. AIDS. 2019;33(9):1455-65.

15. Aloy B, Tazi I, Bagnis $\mathrm{Cl}$, et al. Is Tenofovir alafenamide safer than tenofovir disoproxil fumarate for the kidneys? AIDS Rev. 2016;18(4):184-92.

16. Lalley-Chareczko L, Hiserodt E, Moorthy G, et al. Urine assay to measure tenofovir concentrations in patients taking tenofovir alafenamide. Front Pharmacol. 2020;11:286.

17. Johnson KA, Niu X, Glidden DV, et al. Lower urine tenofovir concentrations among individuals taking tenofovir alafenamide versus tenofovir disoproxil fumarate: implications for point-of-care testing. Open Forum Infect Dis. 2021;8(7):ofab200.

18. Custodio JM, Fordyce M, Garner W, et al. Pharmacokinetics and safety of tenofovir alafenamide in HIV-uninfected subjects with severe renal impairment. Antimicrob Agents Chemother. 2016;60(9):5135-40.

19. Jose S, Hamzah L, Campbell $L$, et al. Incomplete reversibility of estimated glomerular filtration rate decline following tenofovir disoproxil fumarate exposure. J Infect Dis. 2014;210(3):363-73.

20. Nishijima T, Mutoh Y, Kawasaki Y, et al. Cumulative exposure of TDF is associated with kidney tubulopathy whether it is currently used or discontinued. AIDS. 2018:32(2):179-88.

21. Koteff J, Borland J, Chen S, et al. A phase 1 study to evaluate the effect of dolutegravir on renal function via measurement of iohexol and 
para-aminohippurate clearance in healthy subjects. Br J Clin Pharmacol. 2013;75(4):990-6.

22. Milburn J, Jones R, Levy JB. Renal effects of novel antiretroviral drugs. Nephrol Dial Transplant. 2017;32(3):434-9.

23. Yukawa $S$, Watanabe $D$, Uehira T, et al. Clinical benefits of using inulin clearance and cystatin C for determining glomerular filtration rate in HIV-1-infected individuals treated with dolutegravir. J Infect Chemother. 2018;24(3):199-205.

24. Lindeman TA, Duggan JM, Sahloff EG. Evaluation of serum creatinine changes with integrase inhibitor use in human immunodeficiency virus-1 infected adults. Open Forum Infect Dis. 2016;3(2):ofw053.

25. Walmsley SL, Antela A, Clumeck N, et al. Dolutegravir plus abacavirlamivudine for the treatment of HIV-1 infection. N Engl I Med. 2013;369(19):1807-18

26. Raffi F, Rachlis A, Stellbrink HJ, et al. Once-daily dolutegravir versus raltegravir in antiretroviral-naive adults with HIV-1 infection: 48 week results from the randomised, double-blind, non-inferiority SPRING-2 study. Lancet. 2013;381 (9868):735-43.

27. Eron JJ, Clotet B, Durant J, et al. Safety and efficacy of dolutegravir in treatment-experienced subjects with raltegravir-resistant HIV type 1 infection: 24-week results of the VIKING Study. J Infect Dis. 2013;207:740-8.

28. Venter WDF, Moorhouse M, Sokhela S, et al. Dolutegravir plus two different prodrugs of tenofovir to treat HIV. N Engl J Med. 2019;381(9):803-15

29. O'Shaughnessy MM, Hogan SL, Thompson BD, et al. Glomerular disease frequencies by race, sex and region: results from the International Kidney Biopsy Survey. Nephrol Dial Transplant. 2018;33(4):661-9.
30. Pesce F, Schena FP. Worldwide distribution of glomerular diseases: the role of renal biopsy registries. Nephrol Dial Transplant. 2010;25(2):334-6.

31. Feehally J, Floege J. Immunoglobulin a nephropathy and IgA vasculitis (Henoch-Schönlein Purpura). In: Feehally J, Floege J, Tonelli M, Johnson RJ, editors. Comprehensive clinical nephrology. 6th ed. Amsterdam: Elsevier; 2018.

32. Moriyama T, Tanaka K, Iwasaki C, et al. Prognosis in IgA nephropathy: 30-year analysis of 1012 patients at a single center in Japan. PLOS ONE. 2014;9(3):e91756

33. D'Amico G. Natural history of idiopathic IgA nephropathy: role of clinical and histological prognostic factors. Am J Kidney Dis. 2000;36(2):227-37.

34. Barbour SJ, Espino-Hernandez G, Reich HN, et al. The MEST score provides earlier risk prediction in IgA nephropathy. Kidney Int. 2016;89(1):167-75.

35. Working Group of the International Ig ANN, the Renal Pathology S, Cattran DC, et al. The Oxford classification of IgA nephropathy: rationale, clinicopathological correlations, and classification. Kidney Int. 2009; 76(5):534-45.

36. Hamzah L, Jose S, Booth JW, et al. Treatment-limiting renal tubulopathy in patients treated with tenofovir disoproxil fumarate. J Infect. 2017;74(5):492-500

\section{Publisher's Note}

Springer Nature remains neutral with regard to jurisdictional claims in published maps and institutional affiliations.
Ready to submit your research? Choose BMC and benefit from:

- fast, convenient online submission

- thorough peer review by experienced researchers in your field

- rapid publication on acceptance

- support for research data, including large and complex data types

- gold Open Access which fosters wider collaboration and increased citations

- maximum visibility for your research: over $100 \mathrm{M}$ website views per year

At BMC, research is always in progress.

Learn more biomedcentral.com/submissions 